\title{
Uma Linha de Produto de Software baseada na Web Semântica para Sistemas Tutores Inteligentes
}

\author{
Title: Semantic Web-Based Software Product Line for Building Inteligent Tutoring Systems
}

\author{
Alan Pedro da Silva \\ Departamento de Sistemas e Computação \\ Universidade Federal de Campina Grande \\ alanpedro@dsc.ufcg.edu.br
}

\author{
Evandro de Barros Costa \\ Ig Ibert Bittencourt \\ Instituto de Computação \\ Universidade Federal de Alagoas \\ \{evandro,ig.ibert\}@ic.ufal.br
}

\begin{abstract}
Resumo A presente pesquisa aborda a problemática envolvida na concepção e desenvolvimento em larga escala de Sistemas Tutores Inteligentes sob a perspectiva da Web Semântica. Trata-se de um tema inerentemente interdisciplinar, envolvendo, neste trabalho, uma conjunção de aspectos relacionados a Inteligência Artificial, Web Semântica e Engenharia de Software, aplicados aos ambientes educacionais em pauta. No presente trabalho, portanto, propõe-se um estudo sobre a construção efetiva de Sistemas Tutores Inteligentes inseridos como parte de ambientes virtuais de aprendizagem para educação a distância ou presencial, tendo em consideração principalmente características de desenvolvimento baseado em reuso e em larga escala. Nesse sentido, realizou-se uma investigação sobre a adequação das abordagens existentes para este propósito, em conjunto com a definição de uma arquitetura com os componentes essenciais de Sistemas Tutores Inteligentes. Esta arquitetura foi utilizada para especificar uma linha de produto que levou em consideração (i) os diferentes perfis de usuários, (ii) o fato que estes usuários não possuem conhecimento avançado em relação a computação, (iii) a heterogeneidade dos domínios de conhecimento que este tipo de sistema irá manipular e (iv) a constante mudança e evolução do objeto que este tipo de sistema manipula: $\mathrm{O}$ conhecimento. Dessa forma, atendendo as quatro considerações mencionadas, foi desenvolvida e testada uma linha de produto de software baseada na web semântica. Os resultados desta abordagem mostraram-se favoráveis na experimentação realizada em dois domínios diferentes, mostrando-se principalmente sua efetividade relativamente aos aspectos larga escala, adaptatividade e evolução.

Palavras-Chave: Linhas de Produto de Software, Web Semântica, Sistemas Tutores Inligentes
\end{abstract}

This research describes an approach for design and development of Intelligent Tutoring Systems under the Semantic Web perspective, considering aspects of both effectiveness and large scale. It deals with a clearly interdisciplinary theme which combines characteristics of Artificial Intelligence, Semantic Web and Software Engineering, applied to this kind of educational system. Thus, this work proposes a study for the rapid development of Intelligent Tutoring Systems as part of Virtual Learning Environments for presencial or distance education, taking into account characteristics such as reuse and large scale development. In this sense, an assessment of the adequateness of current ITS approaches has been done, as well as the definition of an architecture containing the Intelligent Tutoring Systems essential components. The proposed architecture has been used to specify a product line that takes into consideration (i) the different user profiles, (ii) the nonpossession of advanced computing knowledge by the users (iii) the heterogeneity of knowledge domains that kind of systems will handle and $(v)$ the constant change and evolution of the knowledge manipulated of this kind of systems. This way, satisfying the four mentioned considerations, a software product line based on the Semantic Web was developed and tested. The experiments carried out to evaluate the proposed approach, in two different domains, demonstrating its is effectivenees in terms of large scale, adaptivity and evolution. Keywords: Software Product Line, Semantic Web, Intelligent Tutoring System 


\section{Introdução}

O ponto de partida para esta pesquisa foi uma reflexão sobre como a construção rápida e efetiva de Sistemas Tutores Inteligentes poderia ser viabilizada em uma perspectiva de larga-escala, procurando atender necessidades tanto do desenvolvedor quanto do autor/professor. Nesse sentido, estudaram-se as propostas de abordagens existentes na literatura especializada no tema em questão, optando-se pelo investimento na abordagem de Linhas de Produto. Esta abordagem se mostrou importante por oferecer um reuso, também, na configuração dos produtos, proporcionando um mecanismo facilitado na construção destes, de tal forma que usuários sem conhecimentos especializados em computação possam realizar esta tarefa de uma forma autônoma[5][6][9][12]. Entretanto, verificou-se logo em seguida que as abordagens tradicionais para linha de produto de software não respondiam adequadamente ao requisito de evolução. Tal constatação levou a discussão sobre a adoção de um enfoque semântico para linha de produto. Porém, houve antes um investimento em uma linha de produto, desenvolvendo-se, primeiramente, uma arquitetura que contemplasse requisitos importantes destes sistemas. Esta arquitetura foi baseada na experiência de vários trabalhos anteriores no âmbito do grupo de pesquisa Grupo de Otimização da Web - GrOW, tendo sua origem no modelo Mathema[8] e posteriormente na plataforma MASSAYO[5].

Em um segundo momento, pela necessidade de se manipular uma grande quantidade de tipos de conhecimento, que podem ser produzidos por especialistas diferentes, fez-se necessário utilizar tecnologias da Web Semântica. Isto foi importante porque esta tecnologia possibilita um compartilhamento e reuso de conhecimento, de uma maneira formal e sem ambiguidades. Assim, isso possibilita que softwares possam ser construídos de uma forma mais independente do domínio de conhecimento. Portanto, baseado na premissa de se construir sistemas tutores em larga escala, utilizando-se linhas de produto de software, investigou-se como esta abordagem poderia ser utilizada, levando-se em consideração a importante característica da manipulação do conhecimento inerente ao tema central deste artigo. Nesse sentido, constatou-se que as atuais abordagens de linhas de produto não foram exatamente propostas para serem utilizadas em uma perspectiva da web semântica. Por isso, necessitou-se estudar meios para que fosse possível adaptar esta abordagem para este contexto.

Posteriormente, foi realizado um trabalho de descrição semântica, tanto do conhecimento que os tutores necessitam manipular, quanto ao conhecimento dos próprios artefatos da linha de produto. Em seguida, foram implementados os artefatos específicos, de tal forma que produtos poderiam ser instanciados e implementados. Portanto, os sistemas tutores foram testados em duas etapas, com alunos do Instituto Federal de Alagoas (Campus Palmeira dos Índios), em domínios diferentes, para analisar se os tutores produzidos realmente eram satisfatórios, e também identificar e ajustar o uso de tecnologias da web semântica nesse cenário. Com estes trabalhos, foi possível identificar a possibilidade de automatizar o processo de construção, configuração e adaptação da linha de produto e dos seus produtos aos diferentes domínios. Em paralelo, alguns resultados foram alcançados, tais como:

$[1][7][13][14][15]$.

O restante deste artigo está estruturado do seguinte modo. $\mathrm{Na}$ Seção 2, destacam-se as questões de pesquisa norteadoras deste trabalho, tratando-se de aspectos na construção e evolução de sistema tutores inteligentes. Na Seção 3, apresentam-se os objetivos. Na Seção 4 descrevem-se os modelos desenvolvidos durante a elaboração deste trabalho. Na Seção 5, mostra-se o modelo de feature desenvolvido. Na Seção 6 encontra-se a arquitetura do mecanismo de evolução. Na Seção 7, discute-se a avaliação da abordagem proposta através de dois estudos de caso. Na Seção 8 está descrita a avaliação da linha de produto desenvolvida. Por fim, na Seção 9 apresenta-se a conclusão.

\section{Questões de Pesquisa}

Construir sistemas tutores inteligentes para um domínio de conhecimento qualquer pode ser uma tarefa bastante especializada. Isso acontece porque cada domínio pode possuir características bastante diferentes. Por exemplo, uma simulação pode ser algo interessante para o ensino de conhecimento que exige um nível de abstração alto, tais como programação e física. Por outro lado, no ensino de português, pode ser interessante questões discursivas. No entanto, questões objetivas podem ser interessantes para todos os domínios de conhecimento. Além disso, do ponto de vista de avaliação de conhecimento, pode ser que para um mesmo currículo, os alunos sejam avaliados de forma diferente, por exemplo, alunos que estão inseridos na modalidade PROEJA, por exemplo, devem ser avaliados mais pelo seu esforço e avanço, do que propriamente pelo nível de conhecimento ideal, e alunos que estão concorrendo a vestibulares concorridos devem ser avaliados de forma bastante rigorosa. Por isso, podem existir muitos detalhes no momento de se produzir tutores para um determinado propósito, e por isso, deve existir um processo customizado para construção destes sistemas, de tal forma que o mesmo possa ser adequado 
para cada situação que o mesmo poderá ser inserido.

Todavia, como o processo de construção destes tutores é algo caro, produzir tais tutores para cada propósito em específico seria um trabalho ainda maior. Por isso a busca pelo reuso sempre foi uma questão importante na construção de sistemas tutores inteligentes[10][11]. Por outro lado, é desejável que este reuso seja algo disponível para pessoas especializadas, sobretudo para pessoas com conhecimento avançado de computação. Nesse sentido, existem as ferramentas de autoria, que tem o propósito de fornecer um mecanismo simplificado de construção dos sistemas tutores inteligentes. Estas ferramentas tem objetivos de reduzir o esforço computacional na construção dos sistemas tutores inteligentes, mas elas ainda não foram amplamente difundidas na industria. Isso acontece porque tais ferramentas não fornecem a possibilidade de construção rápida dos sistemas tutores inteligentes, pois é necessário um planejamento de como os tutores deverão se comportar, e muitas delas exigem um conhecimento muito especializado da inteligência artificial.

Além disso, estes sistemas têm uma característica importante, eles lidam com conhecimento. Eles lidam intensivamente com conhecimento do estudante, conhecimento pedagógico e o conhecimento a ser ensinado. De forma natural, cada um destes pode ser evoluído constantemente, sendo isto um problema, porque a vida útil destes sistemas pode se tornar curta. Levando-se em consideração que, por padrão, são necessários conhecimentos especializados de computação para se utilizar mecanismos de reuso, o mesmo acontece para viabilizar algum tipo de evolução. Diante do cenário supracitado, as questões de pesquisa embutidas neste artigo foram:

1. Como viabilizar a construção de Sistemas Tutores Inteligentes em larga escala levando-se em consideração as suas variabilidades? Essa é uma questão complexa, porque as atuais abordagens não fornecem um mecanismo de reuso que proporcione uma construção de diferentes sistemas tutores inteligentes, com combinações de requisitos diferentes, no sentido de fornecer adaptações direcionadas aos mais variados cenários de atuação. Essas adaptações atualmente necessitam de ajustes especializados, havendo, muitas vezes, um trabalho de re-engenharia.

2. Como permitir que tal construção seja também adaptativa para os diferentes domínios de conhecimento? Por padrão, a construção de um determinado tutor está acoplada ao domínio de conhecimento que o mesmo está sendo projetado. Assim, se o tutor foi projetado para um determinado domínio, o mesmo terá que ser adaptado para ser utilizado em outro domínio. Levando-se em consideração a grande quantidade de domínios de conhecimento, o custo para se produzir tutores em larga escala poderia ser muito alto.
3. Como permitir a evolução automatizada para esse tipo de sistema? Evoluir um tutor pode ser uma tarefa bastante trabalhosa. Pois cada tutor tem as suas particularidades, e por isso, cada um deles pode se comportar de maneira distinta. Por isso, deve ser levado em consideração o comportamento de cada tutor, e dependendo de como os aprendizes estão se desenvolvendo, a evolução pode ser necessária de uma maneira específica para cada tutor. Por isso, evoluir de forma customizada cada tutor, que já está sendo disponibilizado e utilizado por aprendizes, pode ser uma tarefa muito trabalhosa. Então, é desejável que este trabalho possa ser realizado de maneira automatizada.

\section{Objetivos}

Após as questões de pesquisa abordadas na seção anterior, apresentam-se aqui os objetivos do presente trabalho constituindo na proposição de uma solução viável para a construção de sistemas tutores inteligentes em ambientes educacionais através da combinação de linhas de produto de software e a web semântica. Este objetivo pode ser remetido aos três objetivos específicos seguintes, focalizados em responder às questões anteriormente descritas.

1. Propor uma linha de produto de software para produção de sistemas tutores inteligentes em larga escala. Desta forma, os objetivos específicos são:

1. Fazer um estudo detalhado do domínio;

2. Propor um modelo de features;

3. Propor uma arquitetura para implementação da linha;

4. Implementar a linha;

5. Testar a linha.

2. Propor uma solução computacional baseada na web semântica para construção de STIs em diferentes domínios de conhecimento. Este objetivo está relacionado a questão 2, abordadas na subseção anterior. As questões abordam os problemas do reuso de software, no sentido de adaptação dos produtos para os diferente domínios de conhecimento. Os objetivos específicos são:

1. Propor e/ou reusar ontologias para modelar o conhecimento que precisa ser adaptável aos tutores;

2. Propor e/ou reusar ontologias para modelar artefatos que serão adaptáveis;

3. Propor mecanismos de validação destas ontologias;

4. Propor uma ferramenta para utilização destas ontologias.

3. Propor uma solução computacional para a evolu- 
ção automatizada de STIs. Este objetivo refere-se à questão 3 , e trata a questão da reconfiguração tanto da linha quanto dos produtos, quando novas features surgirem. Os objetivos específicos são:

1. Propor e implementar uma solução para viabilizar a descoberta de novas features;

2. Propor e implementar uma solução para atualizar a linha de produto;

3. Propor e implementar uma solução para atualizar os produtos existentes.

\section{Modelagem de Sistemas Tutores In- teligentes}

O Processo de modelagem de um Sistema Tutor Inteligente está relacionado aos modelos específicos de características gerais de Sistemas Tutores Inteligentes combinado com os modelos necessários para especificação de Linhas de Produto de Software. Dessa forma, quatro modelos foram combinados e compartilhados para que o domínio de Sistemas Tutores Inteligentes fosse adaptado e projetado no sentido de fornecer uma Linha de Produto de Software. Estes modelos foram reproduzidos em ontologias a fim de viabilizar o compartilhamento de conhecimento entre todos eles, viabilizando uma distribuição de forma organizada entre os mesmos, de tal forma que possa haver uma manutenção ou expansão destes de forma desacoplada entre os demais modelos. Além disso, descrevê-los em ontologias permite que algoritmos de software sejam desenvolvidos para suportar todo o processo de construção dos produtos de forma precisa, dado que o significado de cada termo é anotado de uma forma não ambígua. Os modelos anotados e utilizados no processo de construção dos produtos são os seguintes:

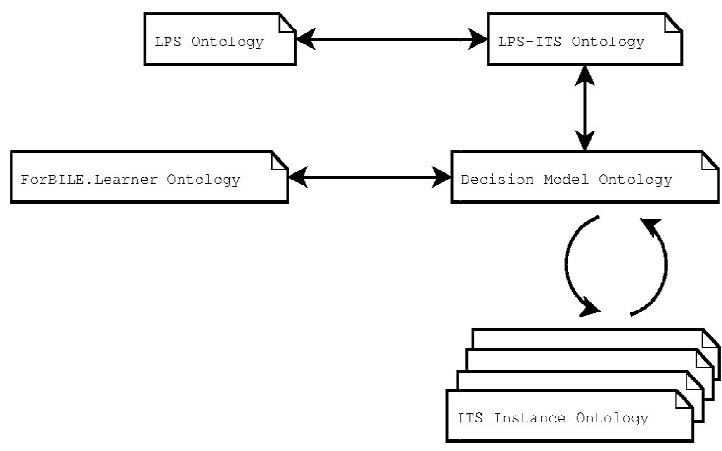

Figura 4.1 Modelo Desenvolvidos

1. LPS Ontology: Ela foi projetada para ser utilizado como um meta-modelo para uma especificação semântica de Feature Oriented Domain Analysis (FODA). A partir deste modelo, é possível modelar ontologias de qualquer domínio de aplicação, dentro das especificações do próprio modelo. Assim, com este modelo separado dos de- mais, foi possível separar as regras específicas de modelagem de linha de produto, que são independentes do domínio que o mesmo é utilizado.

2. LPS-ITS Ontology: Esta especificação segue às definições da Seção 5. Isso é importante porque fornece de forma isolada todas as possibilidades de um sistema tutor, possibilitando que mudanças possam ser feitas de forma independente dos outros modelos, e independente dos domínios de conhecimento do tutor;

3. Forbile.Learner Ontology: Esta ontologia disponibiliza um mecanismo para representação de conhecimento de aprendizes. Assim, este formalismo pode ser compartilhado com outros ambientes ou ferramentas no sentido que funcionalidades possam ser integradas ou adaptadas.;

4. Decision Model Ontology: Esta ontologia representa a instanciação propriamente dita de um Sistema Tutor Inteligente. Com esta ontologia é possível definir as especificidades de cada produto de forma adequada. Isso acontece porque existem regras específicas para este modelo, de tal forma que elas sejam são úteis para a verificação da instanciação dos produtos.

\section{Modelo de Features}

A Figura 5.1 ilustra o modelo de features da linha de produto de sistema tutores inteligente na notação indicada. Para este trabalho, o primeiro passo dentro da modelagem da linha de produto proposta, foram estabelecidas as seguintes relacionamentos entre as features presentes no diagrama representado na Figura 5.1.

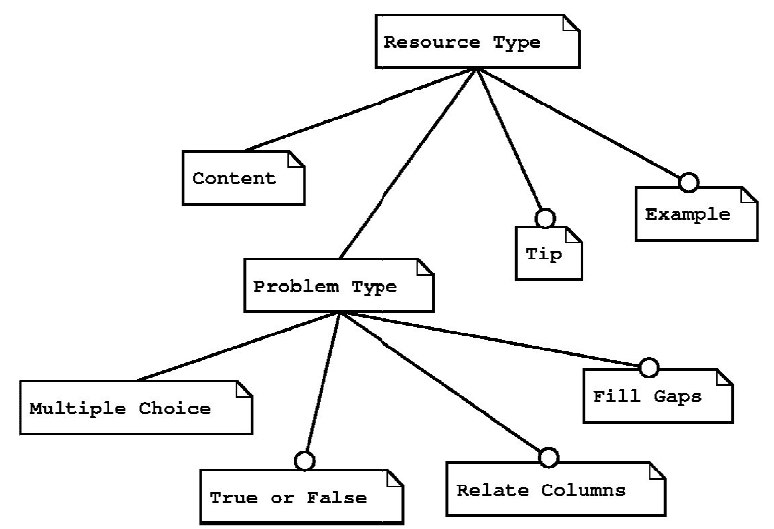

Figura 5.1 Diagrama de Features resumido

Além disso, todos os STIs, desta proposta, contêm obrigatoriamente o método de ensino baseado em resolução de problemas. Contudo, existem variações de como esse método atuará em cada tutor. Essas variação ocorrerão através das escolhas das seguintes features: 
1. General: É uma feature que indica que a explicação de um determinado problema será realizada de maneira geral, ou seja, uma única explicação sobre a teoria envolvida para a resolução de cada problema;

2. Step by Step: Indica que a explicação será realizada através da demonstração de como o problema é resolvido passo a passo. Então, dessa forma, um aprendiz tem a oportunidade de verificar todo o processo da resolução de cada problema.

3. Student Recomendation: É um tipo de explicação onde o estudante tem a possibilidade de entrar em contato com outros estudantes para o ajudar no entendimento da resolução de problema, oferecendo uma perspectiva diferente de explicação.

Também deve ser obrigatório em todo sistema tutor inteligente um modelo do domínio que descreve o domínio que vai ser ensinado para o estudante. Dessa forma, todo modelo do domínio deve conter vários currículos. Além disso, todo curriculum deve possuir recursos. Existem dois tipos de recursos que devem ser obrigatórios para todos os sistemas tutores inteligentes: Conteúdo e Problema. Existem também três tipos de recursos opcionais para as aplicações baseadas na linha de produto: Dica, Exemplo e Modelo do Estudante. Para o tipo de recurso problema, especificamente, o diagrama mostra que o problema do tipo múltipla escolha é obrigatório para todas as aplicações, por outro lado os problemas de verdadeiro ou falso, preencher lacunas e relacionar colunas são opcionais.

\section{Evolução}

Além da descrição semântica dos artefatos, faz-se necessária a implementação de dois recursos que são apropriados para a evolução considerada anteriormente: Componente Semântico e o Meta Componente. Estes dois devem ser incorporados dentro de toda linha (vide Figura 3) descrito abaixo:

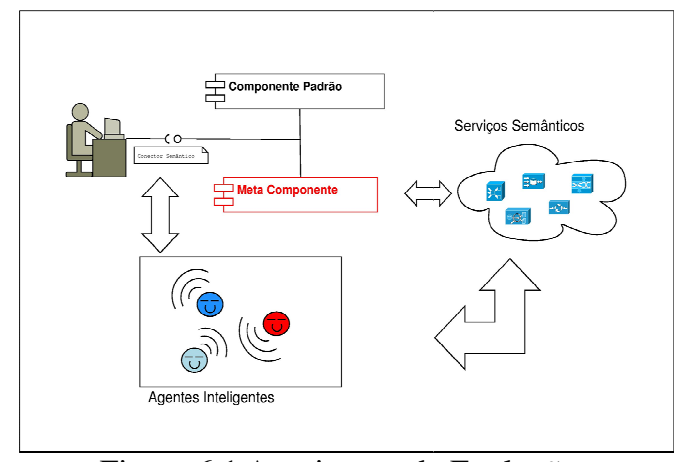

Figura 6.1 Arquitetura da Evolução
1. Componente Tutor: O Componente faz parte dos componentes que são estáticos dentro da arquitetura especificada. Além disso, este componente faz acesso a vários outros componentes para executar as suas tarefas, e neste caso, por exemplo, inicialmente, o componente Conector A. A ideia é que este componente Conector A represente uma determinada feature. No entanto, este componente pode não ser o mais adequado para um determinado perfil de estudante, e por isso, pode ser necessário que o sistema tutor seja reconfigurado para que o componente seja substituído por um outro componente;

2. Conector Semântico: Representa um componente que foi configurado a priori para atender a um determinado conector, no entanto, o mesmo foi estabelecido como um componente possível de mudança automatizada;

3. Ontologia: Assim, para uma eventual mudança automatizada, faz-se necessário que algum mecanismo formal seja utilizado para que agentes de software possam analisar o momento que uma substituição deve ser feita, e além disso, analisar como esta mesma mudança deve ser conduzida;

4. Agentes de Descoberta e Composição de Serviços: Essas mudanças devem ser perceptíveis por agentes de software, e estes mesmo agentes devem identificar se existem novas possibilidades de componentes;

5. Serviços Semânticos: A construção de novos componentes acontece quando novos serviços podem ser utilizados para fornecer alguma funcionalidade variável já especificada no sistema. Isso é importante para que o padrão dos novos componentes seja conhecido pelos agentes computacionais de sentido de fazer com que os mesmos possam identificar com precisão os novos serviços;

6. Meta Componente: Este componente tem por função abstrair o acesso aos serviços semânticos por parte do conector semântico. Então, quando o conector semântico solicita um serviço composto por serviços semânticos, na realidade, uma requisição é enviada ao componente semântico.

\section{Estudo de Caso}

Esta seção tem por objetivo apresentar uma avaliação da abordagem de linha de produto proposta, tendo em conta o investimento em dois domínios: Programação e Física, sendo que apenas o primeiro foi utilizado na descrição seguinte. Para tanto, descreve-se principalmente como os modelos são utilizados com o propósito de construir Sistemas Tutores Inteligentes em Larga Escala. Além disso, está descrito como foram modeladas as instâncias/conceitos nas ontologias de domínio e nas ontologias da linha de produto. 
Ainda, esta Seção descreve como foi construído o Sistema Tutor Inteligente de Programação (o qual será referenciado apenas como tutor no restante deste capítulo). Primeiramente, apresenta-se a modelagem do domínio de programação. Em seguida, descreve-se o processo de instanciação do produto através da ferramenta de autoria. E por fim, demonstra-se como o produto foi implantando.

\subsection{Descriç̃ão do Domínio de Programação}

Nesta seção descreve-se o tutor de programação foi elaborado neste estudo de caso. Dessa forma, fez-se necessário relacionar os aspectos relacionados ao domínio e aos aspectos pedagógicos:

1. Descrição do Domínio: Neste módulo estão as informações a respeito do que será passado para os alunos. No trabalho aqui apresentado o domínio corresponde a assuntos relacionados à programação em linguagem $\mathrm{C}$. $\mathrm{O}$ tutor adota uma abordagem pedagógica de aprendizagem baseada em resolução de problemas, onde os problemas são expressões que podem conter operações, tais como: Entrada de Dados, Saída de Dados, Estrutura de Programa em C, Comando de Atribuição (conforme Figura 7.1.1);

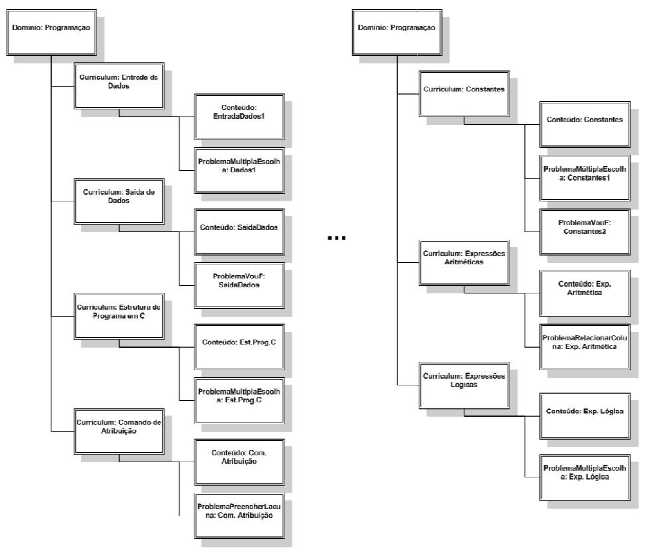

Figura 7.1.1 Descrição do Domínio

2. Definição dos Conteúdos: Conforme as Figuras 7.1.1 e 7.1.2, os conteúdos foram especificados conforme os seus currículos. Então, por exemplo, para o currículo Entrada de Dados, foi especificado um problema múlti-

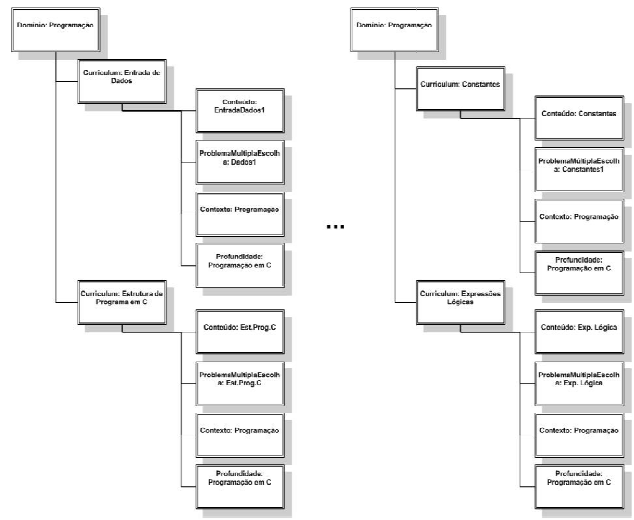

pla escolha (Dados1) e também um foi especificado um conteúdo (EntradaDados1) (conforme Figura 7.1.2);

Figura 7.2.2 Descrição dos Conteúdos

3. Descrição Pedagógica: este módulo faz o gerenciamento do sistema tutor inteligente, tendo a função de coordenar o funcionamento do sistema. Além disso, o mesmo contém estratégias e táticas de ensino, selecionando o conteúdo a ser apresentado ao aluno, monitorando, criticando o desempenho do aluno, além do fornecer assistência quando solicitado. Quando uma questão for proposta para que o estudante a resolva, a interface recebe esta questão através do Módulo Pedagógico que por sua vez obtém do Módulo do Domínio. O Módulo Pedagógico comunica-se diretamente tanto com o Módulo do Domínio quanto com o Modelo do Aprendiz. Essa última comunicação ocorre, por exemplo, quando o usuário solicita a visualização do seu estado de aprendizagem. Desta forma, este módulo deve manter informações sobre a estratégia pedagógica adotada (conforme Figura 7.1.3);

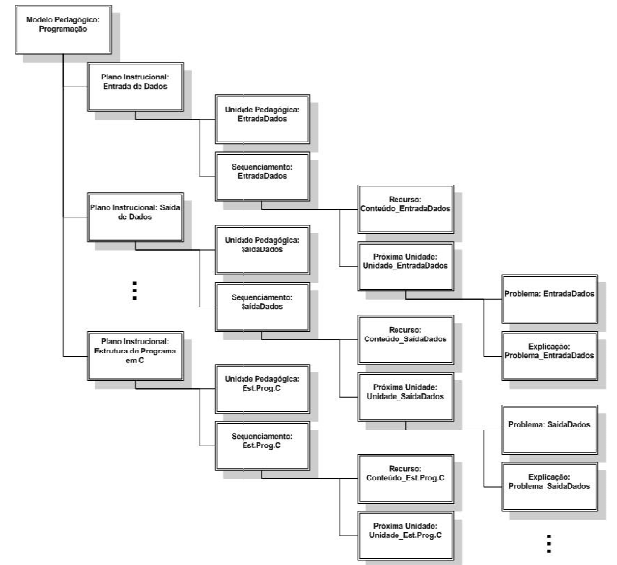

Figura 7.1.3 Descrição Pedagógica

\section{$\underline{7.2 \text { Casos de Uso }}$}

O Cenário pré-estabelecido está descrito na Figura 7.2.1. Nesse cenário, pode-se identificar dois atores, um usuário, ou seja, um aluno eo próprio sistema.

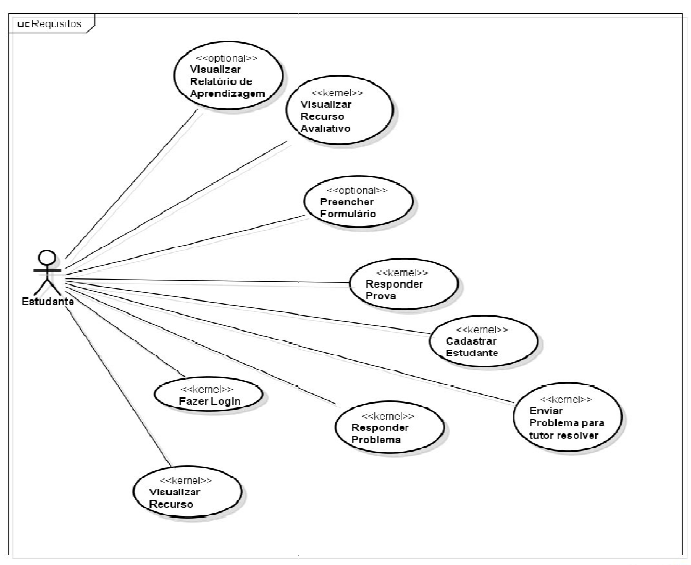


Figura 7.2.1 Casos de Uso Resumido

O estudante é quem faz o seu próprio login no sistema. Isso é importante para que o sistema possa identificar quem é o estudante que está acessando o sistema em um determinado momento; Visualizar Recurso: O estudando também está apto a visualizar recurso. Através disto, o estudante pode passar para um processo de ensinoaprendizagem. Isso ocorre porque são os recursos que proporcionam o ensino aos alunos; Visualizar Recurso Avaliativo: Para que o sistema possa acompanhar de forma mais acurada o nível de conhecimento que o aluno se encontra, faz-se necessário que o mesmo, a cada período, se submeta a Responder Prova, de tal forma que o sistema poderá realizar suas tarefas com mais informação. Além disso, existe o Preencher Formulário, onde está ação é importante para que o sistema possa identificar com mais propriedade o aluno, fazendo-se necessário que o aluno forneça algumas informações iniciais, de tal forma que o sistema poderá mapear com mais precisão o nível de conhecimento que aluno se encontra; Em Cadastrar Estudante encontra-se o processo de cadastro do estudante também é disponibilizado para cada aluno, de tal forma, que o próprio aluno é quem faz o seu cadastro no sistema, colocando as suas informações pessoais de uma forma responsável. Em Resolver Problema, o Aluno também pode responder problemas, isso é importante para que o aluno possa se exercitar, refletindo sobre os seus conhecimentos, para tentar resolver problemas.

O Sistema também possui as suas responsabilidades no processo de ensino aprendizagem. Pois o mesmo precisa acompanhar regularmente cada ação do estudante, e por isso, faz-se necessário que o mesmo também realize suas atividades, tais como:

1. Definir Curriculum para Estudante Interagir: $\mathrm{O}$ Sistema tem a função de definir o currículo para cada estudante. Isso é importante do ponto de vista de personalização, pois cada estudante pode ter níveis de conhecimento diferentes. \%E, se o estudante for submetido a um conhecimento muito além do nível dele, o mesmo poderá ficar desanimado, e da mesma forma que se o estudante se submeter a um conhecimento muito abaixo do nível dele, o mesmo poderá, também, ficar desmotivado;

2. Definir Estratégia Beseada em Teoria de Aprendizagem: Cada estudante pode se adequar a estratégias pedagógicas diferentes de formas diferentes. No entanto, foi definido que o tutor só teria uma única estratégia pedagógica, onde a mesma seria utilizada indistintamente por todos os alunos;

3. Enviar Recurso Avaliativo: Esse tipo de abordagem é interessante, e o sistema pode recorrer ao mesmo quando for necessário identificar com uma melhor quali-

\section{dade o nível do aluno;}

4. Resolver Problema: Existem situações onde o estudante poderá não resolver um determinado problema, mesmo depois dos vários mecanismos de ajuda que o mesmo poderá ser submetido. Dessa forma, em último caso, o sistema poderá ajudar o estudante a resolver um determinado problema;

5. Tutorar: Este procedimento é o mais importante do sistema. Pois o mesmo é responsável por recomendar adequadamente os recursos educacionais aos estudantes. Este procedimento é realizado levando-se em consideração tanto o currículo do estudante, quanto a estratégia pedagógica que o mesmo foi submetido.

\subsection{Diagrama de Features}

Um diagrama de feature proporciona uma visão global da Linha de Produto no que diz respeito as variabilidades e comunalidades da mesma. Assim, torna-se possível perceber os pontos variáveis da arquitetura do sistema, ou melhor, da Linha de Produtos. Com isso, a partir do diagrama geral da Linha de Produto, foi produzido uma linha de produto para tutores de programação. Abaixo segue a figura que exibe o diagrama de feature especifico desta

linha.

Comparando os dois diagramas percebe-se que o STI desenvolvido tem todas as features obrigatórias como previsto. Além do mais, das features opcionais algumas foram providas, tais como:

1. Pedagogical Strategy - Esta está diretamente relacionada a uma questão essencial de um tutor: Como ensinar? Assim, está tem por função proceder dentro do tutor sequenciando recursos educacionais adequadamente para cada perfil de estudante: (i) Primeiramente apresentando conteúdo a cada estudante, (ii) em seguida, depois do estudante ler o conteúdo previamente enviado, o tutor submete um problema adequado para o mesmo;

2. Form: Este recurso é importante para estabelecer um conhecimento inicial sobre cada estudante;

3. True or False Problem: Este é um tipo de problema que é importante para avaliar questões mais pontuais de um determinado currículo;

4. Hint: Este recurso é importante para dar uma dica quando o estudante estiver passando por algum problema, ou para dar uma ajuda inicial quando o estudante não estiver sabendo como proceder para iniciar a resolução de um determinado problema;

5. Domain Learning Report: Este relatório é importante para ajudar os professores a diagnosticar os problemas que estiverem ocorrendo com a turma.

É importante citar que em nível de implementação, cada 
feature foi mapeada em um componente de software. Através disto, pode-se adicionar nova features dinamicamente, sem um custo adicional de implementação em outros componentes.

\subsection{Arquiterura}

A arquitetura definida para o tutor de programação foi a que está expressa na Figura 7.4.1. Nesse sentido, a arquitetura resultante da escolha das features, da subseção anterior, refletem-se basicamente nos cinco pontos de variabilidade abaixo:

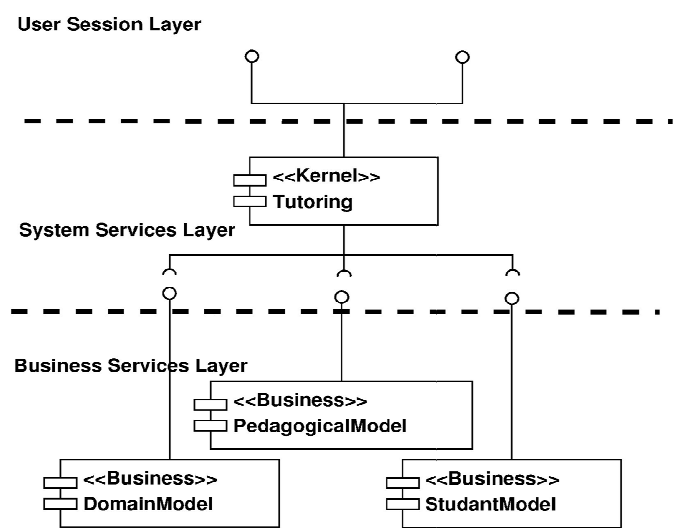

Figura 7.4.1 Arquitetura

Existem alguns tipos de relatórios possíveis, e é possível escolher qualquer combinação deles para ser disponibilizado por um tutor aos seus usuários. Neste caso, foram escolhidos três: Nível de Conhecimento, Performance no Currículo e Nivel de Conhecimento.

Do ponto de vista dos tipos de problemas, também é possível especificar qualquer combinação dos tipos de problemas, No entanto, para o domínio do tutor, foram escolhidos os tipos Múltiplas Escolhas e Falso ou Verdadeiro.

Os recursos avaliativos são meios que o kernel de um tutor tem para avaliar o nível de conhecimento de um determinado aluno. Então, o tutor tem o recurso Teste como obrigatório e o recurso Formulário como opcional.

Já os resolvedores de problemas são importantes e utilizados quando os alunos não conseguem, de uma forma definitiva, resolver um determinado problema que foi submetido a ele. Dessa forma, foi especificado o resolvedor geral de problemas.

Estratégias Pedagógicas: As estratégias pedagógicas são importantes no sentido de proporcionar o recurso educacional correto ao estudante. Nesse sentido, podem ser escolhidas quaisquer combinações, ficando por conta do kernel do tutor definir qual será utilizada por cada estudante.

\section{Avaliação}

Uma avaliação realizada foi baseada em métricas de similaridade para medir a similaridade, a variabilidade e a reutilização da arquitetura de linha de produtos (Tabela 8.1). Essas métricas ajudam a analisar e promover a qualidade da arquitetura da linha de produto. Nesse sentido, as linhas de produto de software contém um conjunto de sistemas de software em um domínio especial. As arquiteturas dos membros da linha devem ser semelhantes, o que é fundamental para a reutilização. Ao medir similaridade, pode-se aperfeiçoar o alcance da linha de produto. Para isto, foi proposto, primeiramente, o Structure Similarity Coefficient (SSC), definido como: (i) Por possuir poucos componentes comuns, e mais componentes variáveis, implicando no $S S C$ baixo, concluímos que a linha de produto apresenta pouca similaridade, perdendo benefícios de reuso. (ii) Notamos que a linha apresenta um VP razoável, se comparamos ao numero total de features 36 , o que implica que pode ser derivado um grande numero de arquiteturas da linha, porém apresenta um nível de complexidade razoável. (iii) Pelo $S C C$ apresentar-se em nível baixo, concluímos que o acoplamento entre os pontos de variabilidade estão em níveis baixos, possuindo assim, pouca dificuldade ao instanciar um PLA. (iv) Com esse, $W C C$, concluímos que a linha de produto não apresenta problemas com relação ao design, uma vez que o valor é baixo. (v) Por possuir um SVC alto, podemos concluir que a linha de produto apresenta uma alta compreensibilidade e mutabilidade.

\begin{tabular}{|c|c|}
\hline \multicolumn{2}{|l|}{ Cálculo das Métricas } \\
\hline Métricas & Valor \\
\hline Número de Features (NF) & 36 \\
\hline Número de principais Features (Ntop) & 6 \\
\hline Profundidade da 'arvore (DT) & 5 \\
\hline Complexidade ciclom'atica (CC) & 1 \\
\hline Restrições Cross-Tree(CTC) & 0,03 \\
\hline Proporção de variabilidade (RoV) & 0,972 \\
\hline Coeficiente de Conectividade/Densidade (CoC) & 1,36 \\
\hline Flexibilidade de configuração (FoC) & 0,53 \\
\hline Número de configurações válidas (NVC) & 524288 \\
\hline Número de features folha (Nleaf) & 23 \\
\hline
\end{tabular}

Tabela 8.1 Cálculo da Métricas

Desse modo, verificou-se a adequação de se propor uma abordagem que combina o uso de Linhas de Produto de Software com serviços Web semânticos, visando proporcionar ambientes que garantam a construção automatiza- 
da de Sistemas Tutores Inteligente em ambientes educacionais sob uma perspectiva de larga escala, levando-se em consideração o reuso e evolução de conhecimento e software.

\section{Conclusão}

Neste trabalho mostrou-se a adequação de se propor uma abordagem que combina o uso de Linhas de Produto de Software com serviços Web semânticos, visando proporcionar ambientes que garantam a construção automatizada de Sistemas Tutores Inteligente em ambientes educacionais sob uma perspectiva de larga escala, levando-se em consideração o reuso e evolução de conhecimento e software.

Um resultado central desta pesquisa foi a constatação de que as linhas de produto de software podem ser utilizadas para aprimorar as atuais abordagens na construção de Sistemas Tutores Inteligentes, em uma perspectiva de larga escala. Sob a perspectiva da web semântica, identificou-se que anotar semanticamente artefatos de software pode reduzir significativamente o esforço relacionado a engenharia de software, sobretudo na modelagem de aspectos cognitivos. Assim, pode-se perceber os seguintes avanços:

A construção em larga escala, de forma customizada de sistemas tutores foi realizada e testada, em uma ambiente escolar. Além disso, o autor de um STI não precisa conhecer aspectos relacionados ao comportamento do sistema, portanto, apenas é necessário conhecer aspectos sobre o domínio atual e as funcionalidades desejadas. Nesse sentido, o esforço e a complexidade para a construção são reduzidos a aspectos relacionados aos requisitos desejados. As ontologias foram a principal ferramenta para isso, porque elas podem representar software e conhecimento. Ademais, as ontologias fornecem um tipo particular de reutilização de software. Isso ocorre porque os artefatos podem ser construídos para diferentes domínios de aplicação, e podem ser utilizados para várias LPS e seus respectivos produtos.

A questão da adaptação dos sistemas tutores aos diferentes domínios é uma característica da modelagem dos tutores e de artefatos anotados semanticamente, pois os mesmos fazem uso de ontologias. Através destas, foi possível separar o conhecimento do software, assim, o software pode ser implementado de forma independente do domínio de sua aplicação. Assim, é possível produzir STI para qualquer domínio com uma mesma implementação.

A evolução automatizada foi viabilizada através do uso de serviços semânticos e agentes, assim, a solução encontrada neste artigo mostra a importância destas tecnologias em sistemas tutores inteligentes. Com isso, novas features podem ser automaticamente adicionadas tanto aos produtos já existentes, quanto a própria linha.

\section{Referências}

[1] Barros, H., Silva, A., Costa, E., Bittencourt, I. I., Holanda, O., and Sales, L. (2011). Steps, techniques, and technologies for the development of intelligent applications based on semantic web services: A case study in e-learning systems. Engineering Applications of Artificial Intelligence, 24(8): 1355 - 1367.

[2] Bittencourt, I. I., Nunes, C., Bezerra, C., de Barros Costa, E., de Oliveira, R. N., Costa, M., Tadeu, M., and da Silva, A. P. (2006). Ontologia para construcão de ambientes interativos de aprendizagem. In Simpósio Brasileiro de Informática na Educação, pages 559-568.

[3] Bittencourt, I. I., Costa, E., Silva, M., and Soares, E. (2009b). A computational model for developing semantic web-based educational systems. Knowledge-Based System, 22(4):302-315

[4] Bittencourt, I. I., Isotani, S., Costa, E., and Mizoguchi, R. (2008c). Researchs directions on semantic web and education. Scientia Journal, 19(1):59-66.

[5] Bittencourt, I. I; Costa, E. (2011). Modelos e Ferramentas para a Construção de Sistemas Educacionais Adaptativos e Semânticos. Revista Brasileira de Informática na Educação. 19(01): 85 - 98.

[6] Bittencourt, I. I., Costa, E., Soares, E., and Pedro, A. (2008b). Towards a new generation of web-based educational systems: The convergence between artificial and human agents. IEEE Multidisciplinary Engineering Education Magazine, 3(1):17-24

[7] da Silva, A. P., Costa, E., Bittencourt, I. I., Brito, P. H. S., Holanda, O., Melo, J., Dermeval, D., and Ribeiro, M. (2010). Ontology-based software product line for building semantic web applications. In In 1st International Workshop on Knowledge-Oriented Product Line Engineering (KOPLE 2010) in conjunction with ACM SIGPLAN International Conference on Systems, Programming, Languages and Applications: Software for Humanity (SPLASH 2010).

[8] Costa, E. B. (1997). Um Modelo de Ambiente Interativo de Aprendizagem Baseado numa ArquiteturaMulti-Agentes. Tese de doutorado, 
Universidade Federal da Paraíba, Campina Grande.

[9] Clements, P. C.; Northrop L. Software Product Lines: Practices and Patterns. SEI Series in Software Engineering. Addison-Wesley, August 2001.

[10] Murray, Tom . Authoring Tools for Advanced Technologies Learning Environments: Toward cost-effective adaptive, interactive and intelligent educational software, chapter An overview of intelligent tutoring system authoring tools: Updated analysis of the state of the art, pages 493-546. Number 17. Kluwer Academic Publishers, Netherlands, 2003.

[11] ParkWoolf, B. Building intelligent interactive tutors : student-centered strategies for revolutionizing e-learning. Morgan Kaufmann Publishers/ Elsevier, Amsterdam ; Boston, 20092009.

[12] Rusk, J.; J., Gasevic, D.. Semantic web services-based reasoning in thedesign of software product lines. In SPLC (2), pages 123-130, 2008.

[13] Silva, A., Costa, E., Bittencourt, I. I., Brito, P., Holanda, O., and Dermeval, D. (2010a). Semantic web-based software product line for building inteligent tutoring systems. In Second International Conference of Software, Services and Semantic Technologies, pages 127-136.

[14] Silva, M., Elias, E., Bittencourt, I. I., Barros, H., Véras, D., Costa, E., and da Silva, A. P. (2010b). Using a model driven approach for specifying educational ontologies. In Proceedings of the 3rd Brazilian Workshop on Semantic Web and Ed

ucation at SBIE'10.

[15] Silva, M., Elias, E., Costa, E., Bittencourt, I. I., Barros, H., da Silva, L. D., da Silva, A. P., and Véras, D. (2011). Combining methontology and a model driven ontology approach to build an educational ontology. IEEE Multidisciplinary Engineering Education Magazine (MEEM). 